\title{
POLIESPLENIA E ANOMALIA DO SISTEMA PORTAL HEPÁTICO ASSOCIADAS À AUSÊNCIA DE VEIA CAVA INFERIOR EM CRIANÇA: RELATO DE CASO
}

\author{
Lucas Cavalcanti dos Santos, Eloisa Nascimento Jorge, Luciana Alvares Calvo, Janilson de Souza Cavalcante \\ Universidade do Oeste Paulista - UNOESTE, Presidente Prudente, SP. e-mail: eloisanjorge@live.com
}

\begin{abstract}
RESUMO
A agenesia da veia cava inferior ( $\mathrm{VCl}$ ) é uma malformação cardiovascular rara que ocorre entre a sexta e décima semana da embriogênese. Pode estar associada a malformações cardíacas e abdominais, além de complicações como a trombose venosa profunda (TVP). O presente trabalho relata o caso de uma paciente diagnosticada ao acaso com agenesia total de $\mathrm{VCl}$ associada à malformação do sistema porto-hepático e poliesplenia. Paciente do sexo feminino, 9 anos, em monitorização por dengue hemorrágica, com tomografia computadoriza e ultrassonografia de abdome revelando anomalia do desenvolvimento do sistema portal hepático associado à ausência de veia cava inferior com continuação na veia ázigos retrocrural, além de múltiplos baços acessórios. Quando as anastomoses das veias primitivas da VCI não se formam adequadamente, pode ocorrer agenesia parcial ou total desse vaso. Consequentemente, o sangue é desviado para a veia ázigos retrocrural. A identificação dessas malformações é importante para que sejam dadas as devidas orientações aos portadores sobre prevenção de TVP, além do acompanhamento desses para monitorização e diagnóstico de outras possíveis malformações.
\end{abstract}

Palavras-chave: veia cava inferior, anormalidades cardiovasculares, malformações vasculares, trombose venosa profunda, poliesplenia.

\section{POLYSPLENIA AND ANOMALY OF THE HEPATIC PORTAL SYSTEM ASSOCIATED WITH THE ABSENCE OF AN INFERIOR VENA CAVA IN A CHILD: A CASE REPORT}

\begin{abstract}
Agenesis of inferior vena cava (IVC) is a rare cardiovascular malformation that occurs between the sixth and tenth week of embryogenesis. It may be associated with cardiac and abdominal malformations, besides complications such as deep vein thrombosis (DVT). The present study reports the case of a patient randomly diagnosed with total IVC agenesis associated with malformation of the portohepatic system and polysplenia. Female patient, 9 years old, being monitored for hemorrhagic dengue, with computed tomography and abdominal ultrasound revealing anomaly of the development of the hepatic portal system associated with the absence of inferior vena cava with continuation in retrocrural azygos vein, in addition to multiple accessory spleens. When the anastomoses of the IVC primitive veins are not formed properly, partial or total agenesis of this vessel may occur. Consequently, the blood is diverted to the retrocrural azygos. Identification of these malformations is important for the patients receive proper guidance about DVT prevention, in addition to monitoring them for diagnosis of other possible malformations.
\end{abstract}

Keywords: inferior vena cava, cardiovascular abnormalities, vascular malformations, deep vein thrombosis, polysplenia. 


\section{INTRODUÇÃO}

As malformações mais frequentes da veia cava inferior ( $\mathrm{VCl}$ ) são a hipoplasia dos segmentos pré-renal, renal e pós-renal e sua duplicidade ${ }^{1}$. A agenesia da $\mathrm{VCl}$ é uma condição cardiovascular ainda mais rara que resulta da ausência do segmento retro-hepático dessa veia, um erro que ocorre durante a embriogênese, entre a sexta e a décima semana de gestação. Na maioria das vezes, está associada a anomalias cardíacas e abdominais².

As anomalias cardíacas mais comuns relacionadas com a agenesia da $\mathrm{VCl}$ incluem dextrocardia, comunicação interatrial e interventricular, estenose da artéria pulmonar ou combinação dessas malformações. Em relação às alterações abdominais, pode ocorrer em associação com transposição de vísceras abdominais e poliesplenia².

A prevalência de malformações da $\mathrm{VCl}$ é de aproximadamente $0,5 \%$, entretanto, pressupõe-se que essa porcentagem seja maior, já que seu diagnóstico geralmente é realizado acidentalmente durante exames de imagem ou cirurgias do abdome, visto que frequentemente é uma condição assintomática ${ }^{2,3}$.

Pacientes portadores de anomalias da $\mathrm{VCl}$ podem apresentar complicações associadas, como a trombose venosa profunda (TVP). Isso ocorre devido a um prejuízo no retorno venoso, ocasionando um aumento na pressão venosa, o que favorece estase sanguínea e formação de trombos ${ }^{4}$. Indivíduos que realizam intensa atividade física, como atletas, e apresentam tais alterações anatômicas da veia cava, possuem maior predisposição para desenvolvimento de TVP, visto que ocorre diminuição na velocidade do fluxo venoso ${ }^{3}$.

O presente trabalho relata um caso de agenesia total de $\mathrm{VCl}$, em paciente assintomática, que foi diagnosticada por exame de imagem devido a complicações de dengue hemorrágica.

\section{RELATO DO CASO}

Paciente do sexo feminino, 9 anos, em acompanhamento pediátrico de rotina em hospital público de Porto Primavera, com histórico de infecção de vias aéreas superiores de repetição, foi encaminhada a um hospital de Presidente Prudente para monitorização em unidade de terapia intensiva por quadro de dengue hemorrágica. Devido a complicações da mesma, foi solicitada ultrassonografia de abdome total, no qual foi identificada veia porta com ramo vascular único adentrando o lobo hepático direito, encurvando-se em direção ao lobo hepático esquerdo (Figura 1).

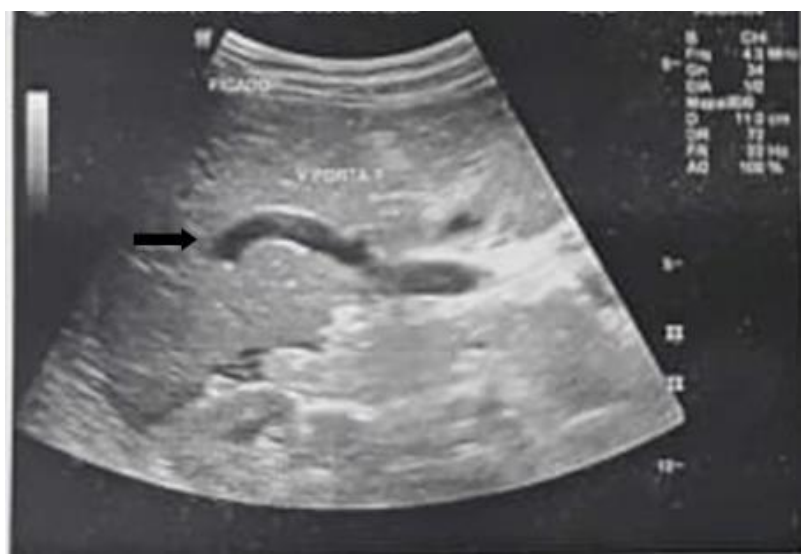

Figura 1. Ultrassonografia de abdome total revelando veia porta com ramo vascular único (seta).

Para melhorar a caracterização da vascularização porto-hepática foi realizada tomografia computadorizada de abdome que identificou sinais de anomalia do desenvolvimento do sistema portal hepático associados à ausência de veia cava inferior com continuação da veia ázigos retrocrural, além de múltiplos baços acessórios (Figura 2).
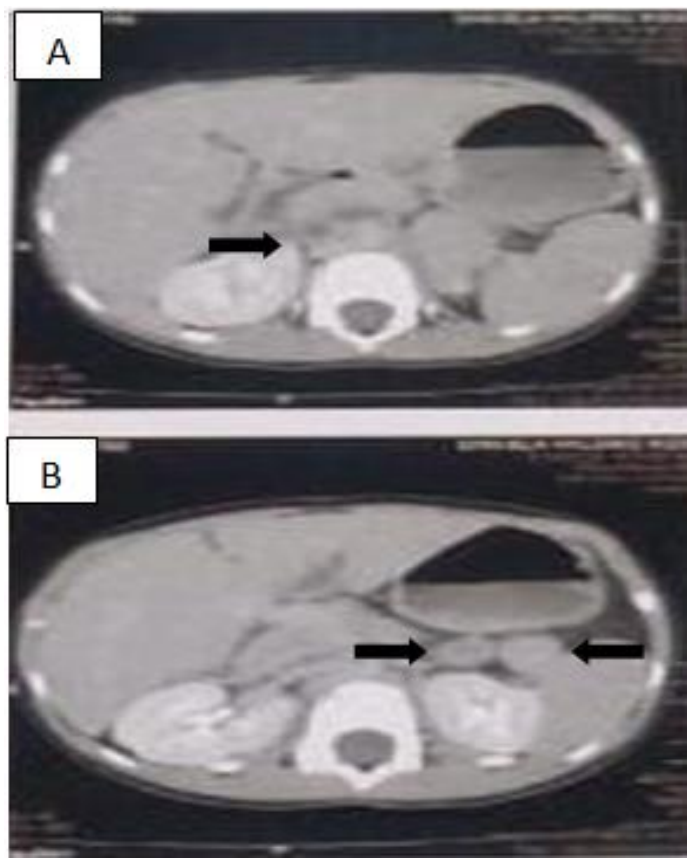

Figura 2. Cortes de tomografia abdominal mostrando a ausência de $\mathrm{VCl}(\mathrm{A}$, seta) e a poliesplenia (B, setas). 
Para complementação diagnóstica foi realizada ultrassonografia de abdome total com doppler do sistema porta, que demonstrou baço com dimensões normais, contornos regulares, ecotextura homogênea, notando-se múltiplas formações nodulares, periesplênicas, medindo até $23 \times 17 \mathrm{~mm}$; anomalia vascular do sistema portal hepático associado a ausência da veia cava inferior (continuação em veia ázigos); hipotrofia do lobo hepático direito e hipertrofia do lobo hepático esquerdo; vesícula biliar em posição anômala, medialmente ao ligamento falciforme (Figura 3).

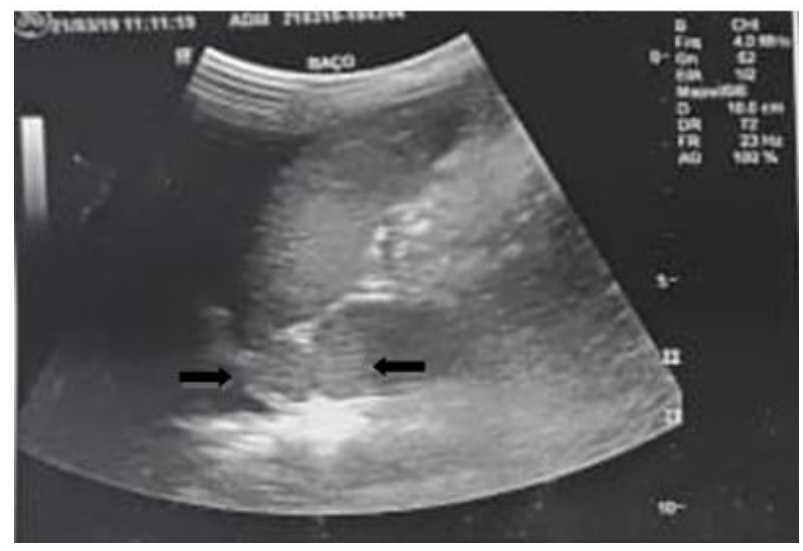

Figura 3. Imagem ultrassonográfica revelando os baços acessórios (setas).

O relato foi submetido ao Comitê de Ética em Pesquisa (CEP) da Unoeste e aprovado sob o protocolo CAAE 25057819.4.0000.5515, estando em concordância com a resolução no 466/2012 do Conselho Nacional de Saúde (CNS).

\section{DISCUSSÃO}

A formação da $\mathrm{VCl}$ é um processo complexo que ocorre entre a $6 \underline{a}$ e a $8^{\underline{a}}$ semana de gestação, o qual coincide com o desenvolvimento do baço, fígado, coração e pulmão. Esse processo envolve as veias póstero-cardinais, subcardinais e supracardinais, as quais desenvolvem extensas anastomoses entre si e depois regridem e atrofiam. A VCl torna-se unilateral, posicionada à direita, no sentido caudal para cranial, nos seus segmentos pós-renal, renal, pré-renal e hepático. Quando ocorre de forma incompleta, esse processo pode resultar em vários tipos de anomalias, como agenesia parcial ou total de $\mathrm{VCl}^{5,6}$.

$\mathrm{Na}$ agenesia total de $\mathrm{VCl}$, embriologicamente, existe uma falha na formação da anastomose subcardino-hepática por volta da 7ạ semana de gestação. Consequentemente, o sangue é desviado para a veia ázigos retrocrural que se encontra dilatada até a croça, juntando-se à veia cava superior no espaço paratraqueal direito ${ }^{7}$. No local onde estaria a $\mathrm{VCl}$ no átrio direito se encontra a veia hepática ${ }^{2}$.

A maioria dos pacientes com malformações da $\mathrm{VCl}$ é assintomática e usualmente o diagnóstico é realizado de forma inesperada por meio de exames de imagem durante a investigação de outras patologias, como no caso relatado, em que a paciente estava sendo monitorizada por dengue hemorrágica ${ }^{8}$.

As anormalidades na formação da $\mathrm{VCl}$ podem ocorrer em associação com transposição de víscera abdominal, disgenesia pulmonar e poliesplenia ${ }^{7}$. A morbimortalidade dos pacientes está relacionada com a gravidade das alterações concomitantes, com piores taxas nos casos de poliesplenia e severas malformações cardíacas ${ }^{9}$.

A agenesia parcial ou total da $\mathrm{VCl}$ promove um aumento do fluxo de drenagem para a circulação colateral abdominal e retroperitoneal como forma de compensação. Contudo, esses vasos não possuem características endoteliais suficientes para suportar o excesso de volume, principalmente em situações de alta demanda metabólica, como no exercício físico. Isso facilita a estase venosa, um dos fenômenos responsáveis pela gênese da TVP ${ }^{10}$. Dessa forma, alguns autores preconizam uso de terapia anticoagulante nesses pacientes, embora não haja consenso definido ${ }^{11}$.

Até o momento da coleta de informações para esse relato, a paciente do caso não apresentou complicações e a condição estava apenas em acompanhamento. Não há indicação de tratamento intervencionista em caso de anomalias de VCI. Entretanto, seu conhecimento é importante para orientar os portadores sobre prevenção de fatores de risco para TVP, como uso de anticoncepcionais orais e longos períodos de imobilização, além de evitar diagnóstico e intervenções errôneas ${ }^{5,12}$.

Apesar de raras, as anomalias congênitas da $\mathrm{VCl}$ podem trazer dificuldades de interpretação de exames de imagem, o que pode prejudicar a tomada de decisões. Dessa forma, a repercussão hemodinâmica e metabólica dessas anomalias e suas possíveis complicações devem ser bem investigadas, para que a melhor estratégia terapêutica seja adotada. 


\section{AGRADECIMENTOS}

Agradecemos aos envolvidos, que permitiram relatar o caso, ao Dr. José Silvio Pettinate, pediatra, e à Universidade do Oeste Paulista pelo auxílio.

\section{CONFLITO DE INTERESSE}

Os autores declaram não haver qualquer potencial conflito de interesse que possa interferir na imparcialidade deste trabalho científico.

\section{REFERÊNCIAS}

1. Faria Neto JP, Miranda RLS. Agenesia de veia cava inferior associada à trombose venosa profunda em jovens. Rev UNINGÁ. 2018;55(1):3541.

2. Felicio LF, Martins AS, Andrade RR, Silva MAM. Ausência parcial de veia cava inferior associada à malformação intestinal. Rev Bras Cir Cardiovasc. 2007;22(3):362-4. DOI: http://dx.doi.org/10.1590/S010276382007000300017.

3. Onzi RR, Costa LF, Angnes RF, Domingues LA, Moraes P, Scaffaro LA. Malformação de veia cava inferior e trombose venosa profunda: fator de risco de trombose venosa em jovens. J Vasc Bras. 2007;6(2):186-9.

DOI: https://doi.org/10.1590/S1677$\underline{54492007000200016}$.

4. Konopka $\mathrm{CL}$, Salame $\mathrm{M}$, Padulla GA, Muradás RR, Batistella JC. Agenesia de veia cava inferior associada à trombose venosa profunda. J Vasc Bras. 2010;9(3):196-9. DOI: http://dx.doi.org/10.1590/S167754492010000300019.

5. Gensas CS, Pires LM, Kruse ML, Leiria TLL, Gomes DG, Lima GG. Agenesia da veia cava inferior. Rev Bras Cardiol Invasiva. 2012;20(4):427-30. DOI: http://dx.doi.org/10.1590/S217983972012000400015.

6. Yun SS, Kim JI, Kim KH, Sung GY, Lee DS, Kim JS et al. Deep venous thrombosis caused by congenital absence of inferior vena cava, combined with hyperhomocysteinemia. Ann Vasc Surg. 2004;18(1):124-9. DOI: https://doi.org/10.1007/s10016-003-0087-x.
7. Kim SJ. Heterotaxy Syndrome. Korean Circ J. 2011;41(5):227-32. DOI: https://doi.org/10.4070/kcj.2011.41.5.227

8. Raymundo SRO, Cabral VS, Cavalieri RF, Neto FR. Thrombolysis for deep venous thrombosis associated with inferior vena cava agenesis in a young patient. BMJ Case Rep 2019;12:e229840. DOI: https://doi.org/10.1136/bcr-2019-229840.

9. Kwon SH, Shin SY. Incidental adult polysplenia with situs inversus, interrupted inferior vena cava with azygos continuation, patent ductus arteriosus, and aortic branches variations: a case report. J Thorac Dis. 2018;10(2):E138-E141. DOI: https://doi.org/10.21037/jtd.2018.01.128.

10. Langer F, Dos Santos D, Suetergaray G, Haygert C. Bilateral deep vein thrombosis associated with inferior vena cava agenesis in a young patient manifesting as low back pain. Acta Med Port 2017;30(4):333-7. DOI: https://doi.org/10.20344/amp.7744.

11. Alves EC, Ferro GBR, França $L K L$, Jacó $M B$, Pitta GBB. Ausência de veia cava inferior: relato de caso. J Vasc Bras. 2010;9(4):254-6. DOI: http://dx.doi.org/10.1590/S167754492010000400010.

12. Yang C, Trad HS, Mendonça SM, Trad CS. Anomalias congênitas da veia cava inferior: revisão dos achados na tomografia computadorizada multidetectores e ressonância magnética. Radiol Bras. 2013;46(4):227-33. DOI: http://dx.doi.org/10.1590/S010039842013000400009. 